\title{
Prolyl Hydroxylase inhibitor repaired chemo-sensitivity through tumor blood vessel normalization in tumor mouse model
}

\author{
Shinji Matsunaga ${ }^{1}$, Shunji Nishide ${ }^{1,2}$, Masayuki Shiota ${ }^{3}$, Takehiro Yamaguchi $^{1}$, Shojiro Kitajima ${ }^{1}$, \\ Katsuyuki Takahashi ${ }^{1}$, Katsuyuki Miura ${ }^{4}$, Shuhei Tomita ${ }^{1}$ \\ ${ }^{I}$ Department of Pharmacology, Osaka City University Graduate School of Medicine, Japan, ${ }^{2}$ Department of Urology, \\ Osaka City University Graduate School of Medicine, Japan, ${ }^{3}$ Division of Research support platform, Osaka City \\ University Graduate School of Medicine, Japan, ${ }^{4}$ Department of Applied Pharmacology and Therapeutics, Osaka City \\ University Graduate School of Medicine, Japan
}

Blood vessel is important tissue structures to deliver oxygen, nutrition and so on. In the tumor tissue, it is often formed abnormal blood vessels which appears hyper-permeability, irregular vascularization, immature vessels and intravasation. This circumstance leads tumor tissue hypoperfusion in accompany with low oxygen and depletion of nutrition. It is called tumor microenvironment which characterized hypoxia, depleted nutrition, low $\mathrm{pH}$ and high interstitial pressure. These environment is one of the causes of chemo- and radio-resistance. In this study, we demonstrated whether Prolyl Hydroxylase (PHD) inhibitor induced blood vessel normalization, improvement of tumor microenvironment and chemotherapy efficiency.

Lewis lung carcinoma cells were subcutaneously transplanted into right frank of mice which aged at 8-12 weeks. Mice were treated with PHD inhibitors intraperitoneally 10 days after the tumor transplant. To assess tumor blood vessels structure and function, endothelial cells were stained for CD31 and analyzed vessel area, diameter and density. Chemosensitivity assessment were performed to analyze apoptosis and DNA damage response, which are used cleaved caspase 3 and gamma H2AX staining respectively, in anti-cancer drug treatment tumors.

PHD inhibitor treatment drastically changed vessel aspects which were increased vessel diameter and decreased CD31 positive area and vessel density. Moreover, pericyte coverage rate were also increased in PHD inhibitor treatment group. PHD inhibitor treatment induced blood vessel normalization in tumor tissues and improved tumor vascular function which are low blood flow, blood leakage and vessel structure. After tumor blood vessel normalization, mice were treated with anti-cancer drugs. Surprisingly, PHD inhibitor and anti-cancer drug treated tumors increased cleaved caspase 3 positive cells and gamma H2AX positive cells and its foci were also increased.

These results suggest that tumor vessel normalization which is induced by PHD inhibitor could lead to not only increase chemo-sensitivity and tissue drug distribution, but also improve efficiency of cancer chemotherapy. It is implied that tumor blood vessel normalization therapy accompanied with angiogenesis is new strategy of cancer therapy. 